\title{
Refinement of Iterative Methods for the Solution of System of Linear Equations Ax=b
}

\author{
Anamul Haque Laskar and Samira Behera \\ Department of Mathematics, Assam University, Silchar, India-788011
}

\begin{abstract}
In this paper we present a survey of three iterative refinement methods for the solution of system of linear equations. The result of this paper shows that the refinement of generalized Jacobi method is much more efficient than the refinement of Jacobi iterative method and is as fast as the refinement of Gauss-Seidel method considering their performance, number of iterations required to converge, storage and level of accuracy. This research will help to appreciate the use of iterative techniques for understanding linear equations.
\end{abstract}

Keywords: Jacobi method, Gauss-Seidel method

\section{Introduction}

The direct methods give the exact solution in which there is no error except the round off error due to the machine, where as iterative methods give the approximate solutions in which there is some error. Basically it gives a sequence of approximation to the solution which converges to the exact solution. The approximate solution does not converge always in nearly singular problems by the use of these methods. The direct methods of solving linear equations have some difficulties. For e.g. the problem of Gaussian elimination method lies in control of the accumulation of rounding errors Turner(1989). This has encouraged many authors like Fridburd et al(1989),Turner(1994),Hageman et al (1998),Forsyth et al(1999), Rajase Keran (1992)to investigate the solutions of linear system of equations by direct and indirect methods. Systems of linear equations arise in a large number of areas both directly in modeling physical situations and indirectly in the numerical solutions of the other mathematical models. These applications occur in all areas of the physical, biological, social science and engineering etc. The linear system problem is, "Given an $n \times n$ nonsingular matrix A and an $n$-vector $b$, the problem is to find an $\mathrm{n}$-vector $\mathrm{x}$ such that $\mathrm{Ax}=\mathrm{b}$ ". The most common source of the above problem is the numerical solution of differential equations. A system of differential equations is normally solved by discretizing the system by means of finite difference methods. The efficiency of any method can be judged by two criteria namely,

(a) how fast it is i.e. how many operations are involved?

(b) how accurate is the computer solution?

Finally, it can be easily realize that direct methods are not appropriate for solving large number of equations in a system when the coefficient matrix is sparse i.e. when most of the elements in a matrix are zero. On the other hand iterative methods are suitable for solving linear equations when the number of equations in a system is very large. Iterative methods are very effective concerning computer storage and time requirements. One of the advantages of using iterative methods is that they require fewer multiplications for large systems. Iterative methods are fast and simple to use when the coefficient matrix is sparse. Also these methods have fewer round off errors as compared to the direct methods. Some of the iterative methods are discussed below:

\section{Refinement Of Jacobi Method}

It is a few modification of Jacobi iterative method. It is the simplest technique to solve a system of linear equations with largest absolute values in each row and column dominated by the diagonal element. In this method the system of equations $A x=b$ is written in the form $x=B x+c$ and the coefficient matrix $A$ is written as $\mathrm{A}=\mathrm{L}+\mathrm{D}+\mathrm{U}$.

The Jacobi method in matrix form as,

$\mathrm{x}^{(\mathrm{k}+1)}=-\mathrm{D}^{-1}(\mathrm{~L}+\mathrm{U}) \mathrm{x}^{(\mathrm{k})}+\mathrm{D}^{-1} \mathrm{~b}$

Now,

$$
\begin{aligned}
& A x=b \\
& \text { or, }(\mathrm{L}+\mathrm{D}+\mathrm{U}) \mathrm{x}=\mathrm{b} \\
& \text { or, } \quad \mathrm{Dx}=-(\mathrm{L}+\mathrm{U}) \mathrm{x}+\mathrm{b} \\
& \text { or, } D x=(D-A) x+b \\
& \text { or, Dx }=\mathrm{Dx}+(\mathrm{b}-\mathrm{Ax}) \\
& \text { or, } x=x+D^{-1}(b-A x)
\end{aligned}
$$

Hence the iterative Jacobi refinement formula in matrix form as,

$$
x^{(k+1)}=x^{(k+1)}+D^{-1}\left(b-A x^{(k+1)}\right) \text {. }
$$


Where, $\mathrm{x}^{(\mathrm{k}+1)}$ appeared in the R.H.S. is given in (1).

We start the iterative procedure for refinement of Jacobi method by choosing the initial approximation and then substitute the solution in the equation (1). After getting the values of x's, we substitute these in the equation (2). In the refinement of Jacobi method we can't use most recently available information and in the next step we can use recently calculated value. We shall continue doing iterations until residual difference is less than pre-defined tolerance. But this method will converge iff the coefficient matrix is diagonally dominant. This method will also might converge even if the above criteria is not satisfied.

\section{Refinement Of Gauss-Seidel Method}

It is also a few modification of Gauss-Seidel iterative method and it is mainly an iterative method used to solve a system of linear equations. The system of equations $A x=b$, where $A$ is a square matrix, $x$ is a vector of unknowns and $b$ is a vector of right hand side values, can be written as, $x=B x+c$ and the coefficient matrix $A$ is written as, $\mathrm{A}=\mathrm{L}+\mathrm{D}+\mathrm{U}$.

The Gauss-Seidel method in matrix form as

Again,

$$
\mathrm{x}^{(\mathrm{k}+1)}=-(\mathrm{D}+\mathrm{L})^{-1} \mathrm{Ux} \mathrm{x}^{(\mathrm{k})}+(\mathrm{D}+\mathrm{L})^{-1} \mathrm{~b} \text {. }
$$

or, $\quad(\mathrm{L}+\mathrm{D}+\mathrm{U}) \mathrm{x}=\mathrm{b}$

or, $(\mathrm{D}+\mathrm{L}) \mathrm{x}=-\mathrm{Ux}+\mathrm{b}$

or, $\quad(\mathrm{D}+\mathrm{L}) \mathrm{x}=[(\mathrm{D}+\mathrm{L})-\mathrm{A}] \mathrm{x}+\mathrm{b}$

or, $(\mathrm{D}+\mathrm{L}) \mathrm{x}=(\mathrm{D}+\mathrm{L}) \mathrm{x}+(\mathrm{b}-\mathrm{Ax})$

or, $\mathrm{x}=\mathrm{x}+(\mathrm{D}+\mathrm{L})^{-1}(\mathrm{~b}-\mathrm{Ax})$

hence, the iterative Gauss-Seidel refinement formula in matrix form as, $\mathrm{x}^{(\mathrm{k}+1)}=\mathrm{x}^{(\mathrm{k}+1)}+(\mathrm{D}+\mathrm{L})^{-1}\left(\mathrm{~b}-\mathrm{Ax} \mathrm{x}^{(\mathrm{k}+1)}\right)$.

where, $\mathrm{x}^{(\mathrm{k}+1)}$ appeared in the R.H.S. is given in equation (3).

Next we start the iterative procedure for refinement of Gauss-Seidel method by choosing the initial approximation and then substitute the solution in the equation (3). After obtaining the values of all x's, we substitute these in the equation (4). In the refinement of Gauss-Seidel method, we use the most recent value. We shall continue doing iteration until and unless relative approximate error is less than the pre-specified tolerance. This method is convergent when the coefficient matrix $\mathrm{A}$ is strictly diagonally dominant and a matrix $\mathrm{A}=\left(a_{i j}\right)_{\mathrm{n} \times \mathrm{n}}$ is said to be diagonally dominant if $\left|a_{i i}\right|>\sum_{j=1, j \neq i}^{n}\left|a_{i j}\right| ; i=1,2,3 \ldots \ldots$.

We shall stop the computation process when this difference between any two consecutive iteration is less than the pre-specified tolerance. Moreover, this method is very much effective concerning computer storage and time requirements. It is fast and simple. It starts with an approximate answer. It is necessary for the refinement of Gauss-Seidel method to have non-zero elements on the diagonal.

\section{Refinement Of Generalized Jacobi Method}

Generalized Jacobi method is a few modification of Jacobi iterative method and refinement of generalized Jacobi method is similarly a few modification of generalized Jacobi iterative method. It is a method with a few computations. In many applications, one faces with the problem of solving large sparse linear systems of the form $\mathrm{Ax}=\mathrm{b}$

where $\mathrm{A}$ is a given non-singular real matrix of order $\mathrm{n}$ and $\mathrm{b}$ is a given $\mathrm{n}$-dimensional vector.

Consider the linear system of equations (5) and splitting made by Davod K. Salkuyeh [3] as,

$$
\mathrm{A}=\mathrm{T}_{\mathrm{m}}+\mathrm{E}_{\mathrm{m}}+\mathrm{F}_{\mathrm{m}}
$$

Where $T_{m}=\left(a_{i j}\right)$ be a banded matrix with band length $2 m+1$ is defined as

$\mathrm{t}_{\mathrm{ij}}=\mathrm{a}_{\mathrm{ij}},|\mathrm{j}-\mathrm{i}| \leq \mathrm{m}$

$$
\begin{aligned}
& \text { And } 0 \text {, otherwise } \\
& \mathrm{x}^{(\mathrm{k}+1)}=-\mathrm{T}_{\mathrm{m}}^{-1}\left(\mathbf{E}_{\mathrm{m}}+\mathrm{F}_{\mathrm{m}}\right) \mathrm{x}^{(\mathrm{k})}+\mathrm{T}_{\mathrm{m}}{ }^{-1} \mathrm{~b} \text {. } \\
& \text { Again } \quad \mathrm{Ax}=\mathrm{b} \\
& \text { or, }\left(\mathrm{T}_{\mathrm{m}}+\mathrm{E}_{\mathrm{m}}+\mathrm{F}_{\mathrm{m}}\right) \mathrm{x}=\mathrm{b} \\
& \text { or, } \mathrm{T}_{\mathrm{m}} \mathrm{x}=-\left(\mathrm{E}_{\mathrm{m}}+\mathrm{F}_{\mathrm{m}}\right) \mathrm{x}+\mathrm{b} \\
& \text { or, } \mathrm{T}_{\mathrm{m}} \mathrm{x} \quad=\left(\mathrm{T}_{\mathrm{m}}-\mathrm{A}\right) \mathrm{x}+\mathrm{b} \\
& \text { or, } \mathrm{T}_{\mathrm{m}} \mathrm{x} \quad=\mathrm{T}_{\mathrm{m}} \mathrm{x}+(\mathrm{b}-\mathrm{Ax}) \\
& \text { or, } \mathrm{x} \quad=\mathrm{x}+\mathrm{T}_{\mathrm{m}}^{-1}(\mathrm{~b}-\mathrm{Ax})
\end{aligned}
$$

And $E_{m}, F_{m}$ are strictly lower and strictly upper triangular parts of $A-T_{m}$ respectively. Then the generalized Jacobi method for solving equation (5) is defined as,

$$
\mathrm{k}=0,1,2,3 \ldots \ldots \ldots
$$

Hence the iterative refinement of generalized Jacobi method in matrix form as,

$$
\mathrm{x}^{(\mathrm{k}+1)}=\mathrm{x}^{(\mathrm{k}+1)}+\mathrm{T}_{\mathrm{m}}^{-1}\left(\mathrm{~b}-\mathrm{Ax} \mathrm{x}^{(\mathrm{k}+1)}\right)
$$

where $x^{(k+1)}$ appeared in the R.H.S. is given in equation (7). 
After that we start with the iterative procedure for refinement of generalized Jacobi method for choosing the initial approximation and then substitute the solution in equation (7). After obtaining the values of x's from equations (7), we substitute these values in equation (8). We shall continue the iteration process until residual difference is less than pre-specified tolerance. This method will converge when the coefficient matrix is strictly diagonally dominant. Matrix is said to be diagonally dominant if the absolute value of the diagonal element in each row has been greater than or equal to summation of absolute values of rest of elements of that particular row. The iterative process is terminated when the convergence criterion is fulfilled. It possesses less memory when programmed. In each iteration accuracy is improved. It starts with an approximate answer and it is fast and simple to use when the coefficient matrix is sparse. It is not applicable for non-square matrices. But nonsquare matrices can be converted into square matrices by taking pseudo inverse of the matrix. Moreover this method is very much effective concerning computer storage and time requirements.

\section{Convergence Of Iterative Methods}

The iteration $\mathrm{x}^{(\mathrm{k}+1)}=\mathrm{Bx}^{(\mathrm{k})}+\mathrm{c}$ converges to the exact solutions with an arbitrary choice of the initial approximation $\mathrm{x}^{(1)}$ iff the matrix $\mathrm{B}^{\mathrm{k}} \rightarrow 0$ as $\mathrm{k} \rightarrow \infty$ i.e., $\mathrm{B}$ is a convergent matrix.

Proof:

We have,

$$
\text { and } \quad \mathrm{x}^{(\mathrm{k}+1)}=\mathrm{Bx}^{(\mathrm{k})}+\mathrm{c} .
$$

subtracting (2) from (1), we get

$$
\mathrm{x}-\mathrm{x}^{(\mathrm{k}+1)}=\mathrm{B}\left(\mathrm{x}-\mathrm{x}^{(\mathrm{k})}\right) \text {..... }
$$

which is true for any value of $\mathrm{k}$.

So, $\quad \mathrm{x}-\mathrm{x}^{(\mathrm{k})}=\mathrm{B}\left(\mathrm{x}-\mathrm{x}^{(\mathrm{k}-1)}\right)$.

Equation (3) \& (4) gives

$$
\mathrm{X}-\mathrm{x}^{(\mathrm{k}+1)}=\mathrm{B}^{2}\left(\mathrm{x}-\mathrm{x}^{(\mathrm{k}-1)}\right)
$$

Proceeding in this way up to k-times, we have $\mathrm{X}-\mathrm{x}^{(\mathrm{k}+1)}=\mathrm{B}^{(\mathrm{k})}\left(\mathrm{x}-\mathrm{x}^{(1)}\right)$

which shows that $\left\{\mathrm{x}^{(\mathrm{k})}\right\}$ converges to the exact solution $\mathrm{x}$ for any arbitrary choice of the initial approximation $\mathrm{x}^{(1)}$ iff $\mathrm{B}^{\mathrm{k}} \rightarrow 0$ as $\mathrm{k} \rightarrow \infty$.

Again when the coefficient matrix $\mathrm{A}=\left(a_{i j}\right)$ is row strictly diagonally dominant matrix i.e.,

$\left|a_{i i}\right|>\sum_{j=1, j \neq i}^{n}|a i j| ; \mathrm{i}=1,2, \ldots \ldots . . \mathrm{n}$

Then clearly the infinity norm of the iteration matrix B is less than 1, i.e., $\|B\|_{\infty}<1$ i.e., B is convergent and hence by the above theorem, the iteration method converges to the exact solution for any arbitrary choice of the initial approximation.

\section{Analysis Of Results}

The efficiency of the three method was compared based on a $6 \times 6$ linear system of equations and is as follows:

$$
\begin{array}{r}
4 \mathrm{x}_{1}-\mathrm{x}_{2}-\mathrm{x}_{4}=1 \\
-\mathrm{x}_{1}+4 \mathrm{x}_{2}-\mathrm{x}_{3}-\mathrm{x}_{5}=0 \\
-\mathrm{x}_{2}+4 \mathrm{x}_{3}-\mathrm{x}_{6}=0 \\
-\mathrm{x}_{1}+4 \mathrm{x}_{4}-\mathrm{x}_{5}=0 \\
-\mathrm{x}_{2}-\mathrm{x}_{4}+4 \mathrm{x}_{5}-\mathrm{x}_{6}=0 \\
-\mathrm{x}_{3}-\mathrm{x}_{5}+4 \mathrm{x}_{6}=0
\end{array}
$$

Results produced by the above system of equations are given in the following table:

\begin{tabular}{|l|c|c|}
\hline \multicolumn{1}{|c|}{ Methods } & Number of iterations & Computer time \\
\hline Refinement of Jacobi method & 10 & 0.45 \\
\hline Refinement of Gauss-Seidel method & 7 & 0.32 \\
\hline Refinement of Generalized Jacobi method & 7 & 0.32 \\
\hline
\end{tabular}

\section{Conclusion}

We have presented the three iterative refinement methods for solving system of linear equations and these are refinement of Jacobi method, refinement of Gauss-Seidel method and the refinement of generalized Jacobi method. One practical example is being studied, i.e., $6 \times 6$ system of linear equations even though the software can accommodate up to $20 \times 20$ system of linear equations. The obtained results shows that the refinement of Jacobi method takes longer time of 0.45 seconds for the $6 \times 6$ system of linear equations. It also takes 10 iterations for the $6 \times 6$ system of linear equations to converge as compared to the other two methods within the same tolerance factor. It will also demand more computer storage to store its data. The other two methods take 0.32 seconds and 0.32 seconds to converge and the number of iterations taken by them are 
respectively 7 and 7. This shows that the refinement of generalized Jacobi and the refinement of Gauss-Seidel method require less computer storage than the other method. Thus the refinement of generalized Jacobi method could be considered more efficient than the refinement of Jacobi method and is as fast as the refinement of Gauss-Seidel iterative method.

\section{References}

[1]. Noreen Jamil, A Comparison of Direct and Indirect Solvers for Linear Systems of Equations. Int. J. Emerg. Sci., 2(2), 310-321, June-2012.

[2]. Ibrahim B. Kalambi, A Comparison of Three Iterative Methods for the Solution of Linear Equations, J. Appl. Sci. Environ. Vol. 12, 2008, no.4, 53-55.

[3]. Davod K. Salkuyeh, Generalized Jacobi and Gauss-Seidel Methods for Solving Linear Systems of Equations, Numer. Math. J. Chinese Uni (Englisher) issue 2, Vol.16: 164-170, 2007.

[4]. V.B. Kumer Vatti and Genanew Gofe Gonfa, Refinement of Generalized Jacobi (RGJ) Method for Solving System of Linear Equations, Int. J. Contemp. Math. Sciences, Vol.6, 2011, no.3, 109-116.

[5]. B. N. Dutta, Numerical Linear Algebra and Applications, Pacific Grove: California (1999).

[6]. D. C. Lay, Linear Algebra and its Applications, New York (1994). 